\title{
FROM APOLLONIAN PACKINGS TO HOMOGENEOUS SETS
}

\author{
Sergei Merenkov and Maria Sabitova \\ University of Illinois at Urbana-Champaign, Department of Mathematics \\ 1409 W. Green Str., Urbana, IL 61801, U.S.A.; merenkov@illinois.edu \\ and The City College of New York, Department of Mathematics \\ Convent Ave. at 138th Str., New York, NY 10031, U.S.A.; smerenkov@ccny.cuny.edu \\ CUNY Queens College, Department of Mathematics \\ 65-30 Kissena Blvd., Flushing, NY 11367, U.S.A.; Maria.Sabitova@qc.cuny.edu
}

\begin{abstract}
We extend fundamental results concerning Apollonian packings, which constitute a major object of study in number theory, to certain homogeneous sets that arise naturally in complex dynamics and geometric group theory. In particular, we give an analogue of Boyd's theorem (relating the curvature distribution function of an Apollonian packing to its exponent and the Hausdorff dimension of the residual set) for Sierpiński carpets that are Julia sets of hyperbolic rational maps.
\end{abstract}

\section{Introduction}

One of the most studied objects in number theory which continues to intrigue mathematicians since ancient times is the theory of Apollonian packings. An Apollonian circle packing can be formed as follows. Consider three mutually exterior-wise tangent circles $C_{1}, C_{2}$, and $C_{3}$ in the plane, i.e., each circle touches each of the other two at exactly one point and the open discs enclosed by $C_{1}, C_{2}$, and $C_{3}$ do not intersect pairwise (e.g., see the circles labeled by 18, 23, and 27 in Figure 1 below). A theorem of Apollonius says that there exist exactly two circles that are tangent to all the three circles $C_{1}, C_{2}$, and $C_{3}$. In our example in Figure 1 it would be the big outer circle, denoted by $C_{0}$, and the small one, say $C_{4}$, inscribed inside the curvilinear triangle formed by arcs of $C_{1}, C_{2}$, and $C_{3}$. Applying the Apollonius theorem to any three mutually tangent circles among $C_{0}, C_{1}, \ldots, C_{4}$ (for the circle $C_{0}$ one should take the open disc it bounds as its exterior), one gets new circles inside the disc bounded by $C_{0}$. Continuing this process indefinitely, one obtains an Apollonian circle packing.

Apollonian circle packings are of interest in number theory in particular due to the observation that if four circles $C_{0}, C_{1}, C_{2}, C_{3}$ have integer curvatures, then all the circles in the packing also have integer curvatures (in Figure 1 the numbers inside circles are their curvatures and $C_{0}$ has curvature 10). There has been extensive research done on number theoretic aspects of Apollonian circle packings as well as on related objects (see [S11] for a summary of recent advances; also [BF11], [G05], [G06-1], [G06-2], [G03], [KO11], [OS12], [S08], and others).

doi:10.5186/aasfm.2015.4046

2010 Mathematics Subject Classification: Primary 52C26, 37F35.

Key words: Apollonian packing, Sierpiński carpet, curvature distribution function, Hausdorff dimension, hyperbolic rational map, Julia set.

The first author was supported by NSF grant DMS-1001144 and the second author was supported by NSF grant DMS-0901230. 


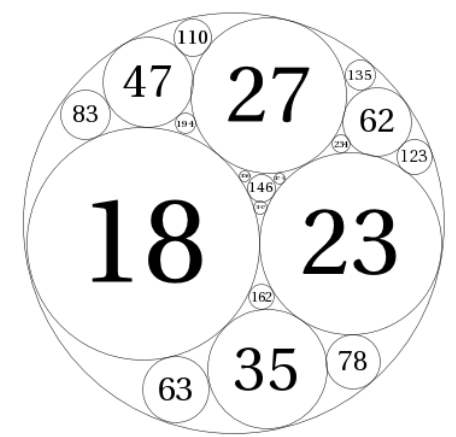

Figure 1. An Apollonian circle packing with integer curvatures.

Consider an Apollonian circle packing $\mathcal{P}$ whose circles are denoted by $C_{0}, C_{1}, \ldots$, and for each $k \in\{0,1, \ldots\}$ let $r_{k}$ denote the radius of $C_{k}$. With $\mathcal{P}$ one associates the exponent $E=E(\mathcal{P})$ given by

$$
E=\inf \left\{t \in \mathbf{R} \mid \sum_{k} r_{k}^{t}<\infty\right\}
$$

Note that $E \neq \pm \infty$. Indeed, clearly $t$ is non-negative, and the series $\sum_{k} r_{k}^{2}$ is convergent since $\pi \sum_{k} r_{k}^{2}$ is bounded by the area enclosed by the outer circle $C_{0}$. One also defines the following curvature distribution function

$$
N(x)=\#\left\{k \mid r_{k}^{-1} \leq x\right\}, \quad x \in \mathbf{R} .
$$

Let $\mathcal{S}$ be the complement of the union of all the open discs enclosed by circles $C_{k}, k \in\{1,2, \ldots\}$, in the closed disc enclosed by $C_{0}$. The set $\mathcal{S}$ is called the residual set of $\mathcal{P}$. It is a set of Lebesgue measure zero and is a fractal in the sense that it behaves similarly on all scales. A natural notion of dimension that can be associated to any metric space, in particular the residual set of an Apollonian circle packing endowed with the restriction of the Euclidean metric, is the Hausdorff dimension. It

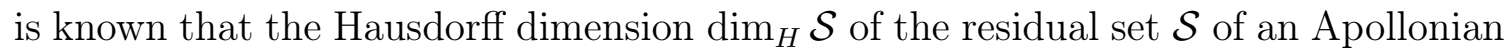
circle packing is approximately 1.305688 (see, e.g., [Mc98, p. 692]). The following result is fundamental in the study of Apollonian circle packings and provides the main motivation for the present paper.

Theorem 1. (Boyd [Bo73, Bo82]) If $\mathcal{P}$ is an Apollonian circle packing, then

$$
\lim _{x \rightarrow \infty} \frac{\log N(x)}{\log x}=E=\operatorname{dim}_{H} \mathcal{S},
$$

where $N$ is the curvature distribution function, $E$ is the exponent of $\mathcal{P}$, and $\operatorname{dim}_{H} \mathcal{S}$ is the Hausdorff dimension of the residual set $\mathcal{S}$ of $\mathcal{P}$.

It is natural to ask whether an analogue of Theorem 1 holds for other subsets of the plane that have fractal nature akin to the residual set of an Apollonian circle packing. In this paper we propose to investigate this question for a family of sets with controlled geometry on all scales, the so-called homogeneous sets defined in Section 2. These are the residual sets of packings by topological (rather than geometric) discs that have roughly rounded shapes, appear at all locations and on all scales, and are relatively separated in the sense that two large elements of the packing cannot be too close to each other. The last condition can be replaced by the assumption that the elements of a packing are uniformly fat. One example of such a homogeneous 
set is the standard Sierpiński carpet $S_{3}$ (see Figure 3 below). For a homogeneous set we establish asymptotic relationships between a certain natural analogue of the curvature distribution function and the Minkowski dimensions of the corresponding residual set $\mathcal{S}$ (Section 3). It turns out that for a general homogeneous set the exponent of the corresponding packing equals the upper Minkowski dimension of $\mathcal{S}$ rather than the Hausdorff dimension of $\mathcal{S}$. As the main application of these results we prove an analogue of Theorem 1 for Sierpinski carpets that are the Julia sets of hyperbolic rational maps (Section 4). Finally, in Section 5 we state some open problems.

Acknowledgments. The authors would like to thank Mario Bonk for many useful comments and suggestions.

\section{Homogeneous sets and carpets}

Let $\left\{C_{k}\right\}_{k \in \mathbf{N} \cup\{0\}}$ be a collection of simple closed curves in the plane such that each $C_{k}, k \in \mathbf{N}$, is enclosed by $C_{0}$ (i.e., $C_{k}$ is contained in the closure of the bounded component $D_{0}$ of the complement of $C_{0}$ ) and suppose that for each pair $j, k \in \mathbf{N}$ with $j \neq k$, the bounded complementary components $D_{j}$ and $D_{k}$ of $C_{j}$ and $C_{k}$, respectively, are disjoint. In analogy with Apollonian circle packings, we call the collection of curves $\mathcal{P}=\left\{C_{k}\right\}_{k \in \mathbf{N} \cup\{0\}}$, or, interchangeably, the collection of the corresponding topological discs $\left\{D_{k}\right\}_{k \in \mathbf{N} \cup\{0\}}$, a packing. The residual set $\mathcal{S}$ associated to such a packing $\mathcal{P}$ (or, simply, the residual set of $\mathcal{P}$ ) is the compact subset of the closure $\bar{D}_{0}$ obtained by removing from $\bar{D}_{0}$ all the domains $D_{k}, k \in \mathbf{N}$. One can similarly define packings and the associated residual sets in the sphere rather than in the plane. In the following it makes no difference whether we consider planar or spherical packings. We say that the residual set $\mathcal{S}$ of a packing $\mathcal{P}$ is homogeneous if it satisfies properties (1), (2), and (3), or (1), (2), and (4) below.

If one hopes to prove an analogue of Theorem 1 for a packing $\mathcal{P}=\left\{C_{k}\right\}_{k \in \mathbf{N} \cup\{0\}}$, then it is reasonable to assume that domains $D_{k}$ 's corresponding to curves $C_{k}$ 's have roughly rounded shapes. More precisely,

(1) there exists a constant $\alpha \geq 1$ such that for each $D_{k}$ there exist inscribed and circumscribed concentric circles of radii $r_{k}$ and $R_{k}$, respectively, with

$$
\frac{R_{k}}{r_{k}} \leq \alpha
$$

For example, (1) holds if all $C_{k}$ 's are circles or squares.

It also seems clear that one needs to impose a condition on the residual set $\mathcal{S}$ associated to $\mathcal{P}=\left\{C_{k}\right\}_{k \in \mathbf{N} \cup\{0\}}$ that imitates the fractal nature of the residual set of an Apollonian circle packing. We consider the following condition, which is standard in complex dynamics and geometric group theory:

(2) there exists a constant $\beta \geq 1$ such that for any $p \in \mathcal{S}$ and $r, 0<r \leq \operatorname{diam} \mathcal{S}$, there exists a curve $C_{k}$ satisfying $C_{k} \cap B(p, r) \neq \emptyset$ and

$$
\frac{1}{\beta} r \leq \operatorname{diam} C_{k} \leq \beta r .
$$

Here and in what follows $B(p, r)$ denotes the open disc of radius $r$ centered at $p$, and $\operatorname{diam} X$ stands for the diameter of a metric space $X$. Geometrically, property (2) means that curves $C_{k}$ 's appear at all locations and on all scales. 
Remark 1. Note that properties (1) and (2) readily imply that the Lebesgue measure of $\mathcal{S}$ is zero. Indeed, otherwise $\mathcal{S}$ would contain a Lebesgue density point, which clearly contradicts to (1) and (2).

Further, we require the following condition of relative separation:

(3) there exists a constant $\delta>0$ such that for any $j \neq k$ we have

$$
\Delta\left(C_{j}, C_{k}\right):=\frac{\operatorname{dist}\left(C_{j}, C_{k}\right)}{\min \left\{\operatorname{diam} C_{j}, \operatorname{diam} C_{k}\right\}} \geq \delta .
$$

Here $\operatorname{dist}\left(C_{j}, C_{k}\right)$ stands for the distance between $C_{j}$ and $C_{k}$. One can visualize property (3) as forbidding two large curves $C_{j}$ and $C_{k}$ to be quantitatively too close to each other.

Remark 2. While extending analysis from Apollonian circle packings to more general packings, one would like to recoup an important property of rigidity. Conditions (1), (2), and (3) can be considered as a way of doing so.

Clearly, Apollonian circle packings satisfy (1). However, they do not satisfy either (2) or (3). Indeed, the failure of (3) is immediate because in Apollonian circle packings the circles are allowed to touch. We now show that (2) fails. Let $C_{1}$ and $C_{2}$ be two tangent circles in an Apollonian circle packing having disjoint interiors and let $p$ denote the common point of $C_{1}$ and $C_{2}$. Assume $\epsilon_{k}$ is a sequence of positive numbers with $\epsilon_{k} \rightarrow 0$ as $k \rightarrow \infty$. For each $k$ denote by $r_{k}$ the radius of a circle from the packing other than $C_{1}$ or $C_{2}$ that intersects $B\left(p, \epsilon_{k}\right)$ and has the largest radius. It can be checked that the sequence $r_{k}$ goes to zero faster than $\epsilon_{k}$ when $k \rightarrow \infty$ (in fact, $r_{k}$ is majorized by $\epsilon_{k}^{2}$ ). Thus homogeneous sets do not generalize Apollonian circle packings but rather complement them.

As pointed out in the previous paragraph, condition (3) does not allow for two elements of a packing to touch. It turns out that in the main results of the paper this condition can be replaced by the following co-fatness condition for $\mathcal{S}$ (see [S95]):

(4) there exists a constant $\tau>0$ such that for every $k$ and each disc $B(p, r)$ that is centered on $D_{k}$ and does not contain $D_{k}$, we have

$$
\operatorname{area}\left(D_{k} \cap B(p, r)\right) \geq \tau r^{2} \text {. }
$$

Remark 3. It is easy to check that neither of (3) or (4) implies the other. Condition (4) is often easier to check, e.g, as (1) it holds in the case when all $C_{k}$ 's are circles or squares. Moreover, the Sierpiński gasket (see Figure 2) satisfies (4) but not (3). It is not hard to see that the Sierpiński gasket also satisfies (1) and (2), and so it is homogeneous.

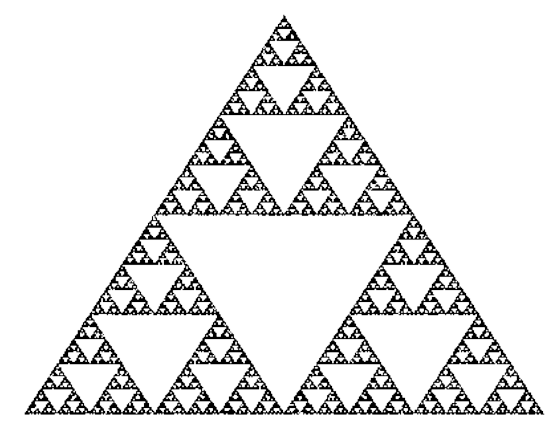

Figure 2. The Sierpiński gasket. 
Example 1. Standard Sierpiński carpets are homogeneous. Recall that for an odd integer $p>0$ the standard Sierpiński $p$-carpet $S_{p}$ (defined up to translations and scalings of the plane) is obtained as follows. As the first step of the construction we subdivide a closed square in the plane into $p^{2}$ equal subsquares in the obvious way and then remove the interior of the middle square (the middle square is well defined because $p$ is odd). In the second step we perform the same operations (subdivide into $p^{2}$ equal subsquares and remove the interior of the middle square) on the $p^{2}-1$ subsquares remaining after the first step. If the process is continued indefinitely, then what is left is called the standard Sierpinski p-carpet (see Figure 3 below for $p=3$ ). A proof that $S_{3}$ and $S_{5}$ are homogeneous is contained in Example 3 below. For a general $p$ the arguments are similar.

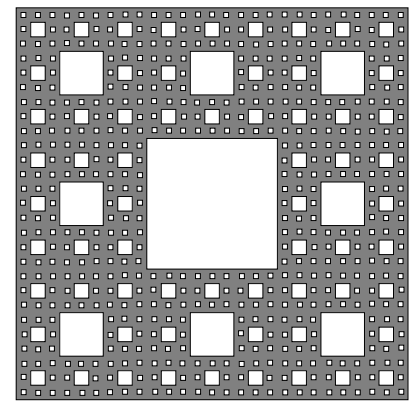

Figure 3. The standard Sierpiński 3-carpet $S_{3}$.

By definition, a Sierpinski carpet, or carpet for short, is any topological space that is homeomorphic to $S_{3}$. A peripheral circle of a carpet $S$ is any simple closed curve in $S$ that corresponds under a homeomorphism to the boundary of one of the removed squares in the construction of $S_{3}$. Whyburn's characterization [W58] states that a metrizable topological space is a carpet if and only if it is a planar continuum of topological dimension 1 that is locally connected and has no local cut points. Here a local cut point is a point whose removal separates the space locally. This gives a way to produce a large supply of carpets as follows. Suppose that $\bar{D}_{0}$ is a closed topological disc in the plane or in the sphere and $\left\{D_{k}\right\}_{k \in \mathbf{N}}$ are pairwise disjoint open topological discs contained in $D_{0}$ that are bounded by simple closed curves. Then the space $\mathcal{S}=\bar{D}_{0} \backslash \cup_{k \in \mathbf{N}} D_{k}$ is a carpet if and only if $\mathcal{S}$ has no interior, $\operatorname{diam} D_{k} \rightarrow 0$ as $k \rightarrow \infty$, and for each pair $j, k \in \mathbf{N} \cup\{0\}, j \neq k$, the boundaries $\partial D_{j}$ and $\partial D_{k}$ are disjoint. In this way, any carpet is represented naturally as the residual set associated to a packing $\mathcal{P}=\left\{\partial D_{k}\right\}_{k \in \mathbf{N} \cup\{0\}}$, where the boundaries of $D_{k}$ 's are peripheral circles. As a partial converse, any homogeneous residual set $\mathcal{S}$ satisfying (3) is a carpet. Indeed, (1) implies diam $D_{k} \rightarrow 0$ as $k \rightarrow \infty$, (2) gives that $\mathcal{S}$ has no interior, and (3) implies that $\partial D_{j}$ and $\partial D_{k}$ are disjoint for all $j \neq k$.

Example 2. If the Julia set $\mathcal{J}(f)$ of a hyperbolic rational map $f$ is a carpet, then it is homogeneous (see the proof of Theorem 6 below; definitions of a Julia set and hyperbolicity can be found in Section 4 below). An example of such a map is $f(z)=z^{2}-1 /\left(16 z^{2}\right)$ (see Figure 4 for its Julia set).

It is known that if the limit set $\mathcal{L}(G)$ of a convex-cocompact Kleinian group $G$ is a carpet, then it is homogeneous. (This can be extracted from [KK00].) In particular, if $G$ is the fundamental group of a compact hyperbolic 3-manifold with non-empty totally geodesic boundary, then its limit set $\mathcal{L}(G)$ is a homogeneous carpet. We do not prove these statements as we do not need them. For a comprehensive treatment 
of asymptotics of the curvature distribution function for circle packings invariant under non-elementary Kleinian groups see [OS12]. For related results on Kleinian groups see [B97] and [P95].

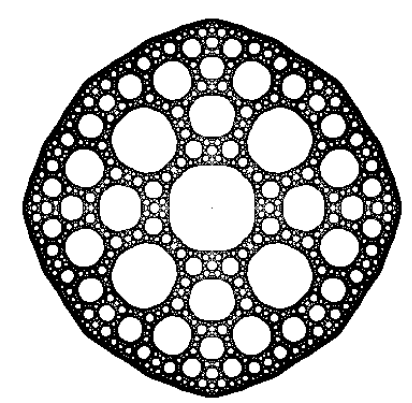

Figure 4. The Julia set of $f(z)=z^{2}-\frac{1}{16 z^{2}}$.

Assume that $\mathcal{P}=\left\{C_{k}\right\}_{k \in \mathbf{N} \cup\{0\}}$ is a packing whose associated residual set $\mathcal{S}$ satisfies (1). Similarly to the case of Apollonian circle packings we define the exponent $E=E(\mathcal{P})$ of $\mathcal{P}$ as

$$
E=\inf \left\{t \in \mathbf{R} \mid \sum_{k}\left(\operatorname{diam} C_{k}\right)^{t}<\infty\right\} .
$$

Let $a_{k}=\operatorname{diam} C_{k}$ and for a natural $m$ denote $M(t, m)=\sum_{k=1}^{m} a_{k}^{t}, M(t)=\sum_{k} a_{k}^{t}$. As in the case of Apollonian circle packings we have $M(2)<\infty$. Indeed, using (1) we have

$$
\pi \sum_{k} a_{k}^{2} \leq 4 \pi \sum_{k} R_{k}^{2} \leq 4 \alpha^{2} \sum_{k} \pi r_{k}^{2} \leq 4 \alpha^{2} \operatorname{Area}\left(D_{0}\right),
$$

since the circles inscribed in $C_{k}$ 's are pairwise disjoint. Also, without loss of generality we can assume that all $a_{k} \leq 1$, and thus for $t \geq t^{\prime} \geq 0$ we have $M(t, m) \leq M\left(t^{\prime}, m\right)$ for any $m$. Finally, $M(0)=\infty$. These imply that $E<\infty$ and

$$
E=\inf \left\{t \in \mathbf{R} \mid \sum_{k}\left(\operatorname{diam} C_{k}\right)^{t}<\infty\right\}=\sup \left\{t \in \mathbf{R} \mid \sum_{k}\left(\operatorname{diam} C_{k}\right)^{t}=\infty\right\} .
$$

The curvature distribution function $N(x)$ of $\mathcal{P}$ is defined by

$$
N(x)=\#\left\{k \mid\left(\operatorname{diam} C_{k}\right)^{-1} \leq x\right\}, \quad x \in \mathbf{R} .
$$

It turns out that for a general homogeneous residual set $\mathcal{S}$ associated to a packing $\mathcal{P}$ the limit of $\log N(x) / \log x$ does not exist when $x$ tends to infinity, i.e.,

$$
\limsup _{x \rightarrow \infty} \frac{\log N(x)}{\log x} \neq \liminf _{x \rightarrow \infty} \frac{\log N(x)}{\log x} .
$$

Also, the Hausdorff dimension of $\mathcal{S}$ is not necessarily equal to $E(\mathcal{P})$ (see Example 3 below). This contrasts Theorem 1.

Example 3. Using the idea of Sierpiński carpets we now construct the following packing for which (5) holds. To a closed square in the plane we apply the first $n_{1}$ steps of the construction of the 3-carpet. Then, to each of the remaining subsquares we apply the first $n_{2}$ steps of the construction of the 5-carpet. After that, to each of the remaining subsquares we apply the first $n_{3}$ steps of the construction of the 3-carpet again. This way, alternating, we continue indefinitely. For each sequence $\sigma=\left\{n_{1}, n_{2}, n_{3}, \ldots\right\}$ we therefore have a packing $\mathcal{P}=\mathcal{P}(\sigma)$ and the associated residual 
set $\mathcal{S}=\mathcal{S}(\sigma)$ depending on $\sigma$. In this construction we allow some $n_{k}$ to be infinite, in which case we assume that the sequence $\sigma$ is finite and its last element is infinity. We also allow the first $n_{1}$ steps to be the steps of the construction of the 5-carpet. Whyburn's characterization implies that $\mathcal{S}$ is a carpet.

We now check that for any $\sigma$ the set $\mathcal{S}=\mathcal{S}(\sigma)$ is homogeneous, namely it satisfies conditions (1), (2), and (3). In fact, it also clearly satisfies (4). Indeed, (1) is trivially satisfied, since each bounded complementary component of $\mathcal{S}$ is a square. To check (2) we fix arbitrary $p \in \mathcal{S}$ and $0<r \leq \operatorname{diam} \mathcal{S}$. After performing a number of steps of dividing and removing subsquares as explained in the previous paragraph we will denote by $Q$ one of the biggest subsquares that turn out to be inside $B(p, r)$. Assume also that $Q$ is not a complementary component, i.e., $Q$ is not a "hole". More precisely, suppose that $n=n_{1} n_{2} \ldots n_{k-1} n_{k}^{\prime}$, where $1 \leq n_{k}^{\prime} \leq n_{k}$, is the smallest number such that one of the remaining subsquares $Q$ of side length $s=3^{-n_{1}} 5^{-n_{2}} \ldots 3^{-n_{k}^{\prime}}$ or $3^{-n_{1}} 5^{-n_{2}} \ldots 5^{-n_{k}^{\prime}}$, depending on whether $k$ is odd or even, is contained in $B(p, r)$. From the minimality of $n$ it is immediate that $r / \beta^{\prime} \leq s \leq \beta^{\prime} r$, where $\beta^{\prime} \geq 1$ is an absolute constant. Now we choose $C$ to be the boundary of the middle square in the subdivision of $Q$. Then

$$
\operatorname{diam} C=\frac{\sqrt{2} s}{3} \text { or } \frac{\sqrt{2} s}{5}
$$

and (2) follows with $\beta=5 \beta^{\prime} / \sqrt{2}$. Finally, to verify (3) let $C_{j}$ and $C_{k}$ be the boundaries of two distinct complementary squares in the construction of $\mathcal{S}$. We may assume that $\operatorname{diam} C_{j} \leq \operatorname{diam} C_{k}$. Let $Q$ be the subsquare in the construction of $\mathcal{S}$ so that $C_{j}$ is the boundary of the middle square in the subdivision of $Q$. Because $\operatorname{diam} C_{j} \leq \operatorname{diam} C_{k}$, the curve $C_{k}$ does not intersect the interior of $Q$ and hence

$$
\Delta\left(C_{j}, C_{k}\right)=\frac{\operatorname{dist}\left(C_{j}, C_{k}\right)}{\operatorname{diam} C_{j}} \geq \frac{\operatorname{dist}\left(C_{k}, \partial Q\right)}{\operatorname{diam} C_{j}} \geq \frac{1}{\sqrt{2}},
$$

i.e., (3) follows with $\delta=1 / \sqrt{2}$. So, any $\mathcal{S}$ in the above construction satisfies (1), (2), (3), and (4) and, in fact, the constants $\alpha, \beta, \delta, \tau$ do not depend on sequence $\sigma$.

If $\mathcal{S}$ is the 3 -carpet, then one can easily check that

$$
\lim _{x \rightarrow \infty} \frac{\log N(x)}{\log x}=\frac{\log 8}{\log 3} .
$$

Likewise, if $\mathcal{S}$ is the 5 -carpet, then

$$
\lim _{x \rightarrow \infty} \frac{\log N(x)}{\log x}=\frac{\log 24}{\log 5} .
$$

It is an elementary exercise to show that there exists a sequence $\sigma$ converging to $\infty$ fast enough so that

$$
\limsup _{x \rightarrow \infty} \frac{\log N(x)}{\log x}=\frac{\log 24}{\log 5} \quad \text { and } \quad \liminf _{x \rightarrow \infty} \frac{\log N(x)}{\log x}=\frac{\log 8}{\log 3}<\frac{\log 24}{\log 5} .
$$

Indeed, given a sequence $\left\{\epsilon_{1}, \epsilon_{2}, \ldots\right\}$ of positive numbers converging to zero it is possible to find a large enough $n_{1}$ so that for any $n_{2}^{\prime}, n_{3}^{\prime}, \ldots$ the curvature distribution function $N(x)$ corresponding to $\mathcal{S}(\sigma), \sigma=\left\{n_{1}, n_{2}^{\prime}, n_{3}^{\prime}, \ldots\right\}$, satisfies $\log N\left(x_{1}\right) / \log x_{1}<$ $\log 8 / \log 3+\epsilon_{1}$ for some large $x_{1}$. Similarly, it is possible to find a large enough $n_{2}$ so that for any $n_{3}^{\prime}, n_{4}^{\prime}, \ldots$ the curvature distribution function $N(x)$ corresponding to 
$\mathcal{S}(\sigma), \sigma=\left\{n_{1}, n_{2}, n_{3}^{\prime}, n_{4}^{\prime}, \ldots\right\}$, satisfies $\log N\left(x_{2}\right) / \log x_{2}>\log 24 / \log 5-\epsilon_{2}$ for some $x_{2}>x_{1}$, and so on. We leave the (easy) details to the reader.

Also, the Hausdorff dimension is always at most the lower Minkowski dimension (see the definition of the latter below). Thus, according to Proposition 2 below for such a sequence $\sigma$ and the corresponding set $\mathcal{S}$ one has

$$
\operatorname{dim}_{H} \mathcal{S} \leq \liminf _{x \rightarrow \infty} \frac{\log N(x)}{\log x}=\frac{\log 8}{\log 3},
$$

where $\operatorname{dim}_{H} \mathcal{S}$ denotes the Hausdorff dimension of $\mathcal{S}$. Theorem 4 below in addition shows that

$$
E(\mathcal{P})=\limsup _{x \rightarrow \infty} \frac{\log N(x)}{\log x}=\frac{\log 24}{\log 5}
$$

\section{Curvature distribution function and Minkowski dimensions}

As we saw in Example 3 above, for a general homogeneous residual set $\mathcal{S}$, i.e., the residual set $\mathcal{S}$ of a packing $\mathcal{P}$ satisfying (1), (2), and (3), or (1), (2), and (4), $E$

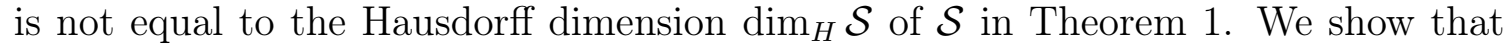
the right analogue is the upper Minkowski (or the upper box counting) dimension of $\mathcal{S}$, which is another notion of dimension applied to fractals. Suppose $n(\epsilon)$ denotes the maximal number of disjoint open discs of radii $\epsilon>0$ centered on $\mathcal{S}$. Then the Minkowski dimension $\operatorname{dim}_{\text {box }} \mathcal{S}$ of $\mathcal{S}$ is defined as

$$
\operatorname{dim}_{\text {box }} \mathcal{S}=\lim _{\epsilon \rightarrow 0} \frac{\log n(\epsilon)}{\log (1 / \epsilon)},
$$

provided the limit exists; see [F14, Ch. 3]. In general, this limit does not exist and in that case one defines the upper Minkowski dimension $\operatorname{dim}_{\mathrm{ubox}} \mathcal{S}$ (respectively, lower Minkowski dimension $\operatorname{dim}_{\text {lbox }} \mathcal{S}$ ) as the corresponding upper limit (respectively, lower limit). Note that there are other equivalent definitions of Minkowski dimensions, e.g., where $n(\epsilon)$ is the minimal number of open discs of radii $\epsilon$ required to cover the set $\mathcal{S}$, or $n(\epsilon)$ is the minimal number of open discs of radii $\epsilon$ centered on $\mathcal{S}$ required to cover $\mathcal{S}$, etc.

The following proposition is a certain duality result between a packing and the associated residual set in the case when the latter is homogeneous.

Proposition 2. If the residual set $\mathcal{S}$ of a packing $\mathcal{P}$ is homogeneous, then

$$
\begin{gathered}
\limsup _{x \rightarrow \infty} \frac{\log N(x)}{\log x}=\operatorname{dim}_{\text {ubox }} \mathcal{S}, \\
\liminf _{x \rightarrow \infty} \frac{\log N(x)}{\log x}=\operatorname{dim}_{\text {lbox }} \mathcal{S},
\end{gathered}
$$

where $N(x)$ is the curvature distribution function of $\mathcal{P}$.

We will need the following lemma.

Lemma 3. Assume that $\mathcal{S}$ is the residual set associated to a packing $\mathcal{P}=$ $\left\{C_{k}\right\}_{k \in \mathbf{N} \cup\{0\}}$ satisfying (1) and (3) (or (1) and (4)). Given any $\beta>0$ there exist constants $\gamma_{1}=\gamma_{1}(\beta) \geq 1$ that depends only on $\beta$ and $\gamma_{2}=\gamma_{2}(\alpha, \beta, \delta) \geq 1\left(\gamma_{2}=\right.$ $\left.\gamma_{2}(\alpha, \beta, \tau) \geq 1\right)$ that depends only on $\alpha$ in (1), $\beta$, and $\delta$ in (3) (that depends only on $\alpha$ in (1), $\beta$, and $\tau$ in (4)) such that for any collection $\mathcal{C}$ of disjoint open discs of radii $r$ centered on $\mathcal{S}$ we have the following. 
(6) There are at most $\gamma_{1}$ discs in $\mathcal{C}$ that intersect any given $C_{k}$ with

$$
\operatorname{diam} C_{k} \leq \beta r
$$

and

(7) there are at most $\gamma_{2}$ curves $C_{k}$ intersecting any given disc in $2 \mathcal{C}$ and satisfying

$$
\frac{1}{\beta} r \leq \operatorname{diam} C_{k},
$$

where $2 \mathcal{C}$ denotes the collection of all open discs with the same centers as those in $\mathcal{C}$, but whose radii are $2 r$.

Proof. By rescaling the Euclidean metric by $1 / r$, we conclude that to find the bound $\gamma_{1}$ in (6) is the same as to find an upper bound on the number of disjoint open discs in the plane of radii 1 that intersect a given set $S$ of diameter at most $\beta$. If $B$ is a disc centered at a point of $S$ and whose radius is $\beta+2$, then $B$ contains $S$ as well as any disc of radius 1 that intersects $S$. Therefore, if $n$ is the number of disjoint open discs of radii 1 that intersect $S$, then by comparing areas we obtain $n \leq(\beta+2)^{2}$.

To prove (7) we first observe that (3) or (4) imply the existence of constants $\beta^{\prime}>0$ and $\nu \in \mathbf{N}$ such that the number of $C_{k}$ 's that intersect a given $B(p, 2 r) \in 2 \mathcal{C}$ and $\operatorname{diam} C_{k}>\beta^{\prime} r$ is at most $\nu$. Indeed, if (3) holds, let $\beta^{\prime}=4 / \delta$ and suppose that there are two distinct curves $C_{j}, C_{k}$ that intersect $B(p, 2 r)$ and $\min \left\{\operatorname{diam} C_{j}\right.$, $\left.\operatorname{diam} C_{k}\right\}>$ $\beta^{\prime} r$. Then $\operatorname{dist}\left(C_{j}, C_{k}\right) \leq 4 r$ and hence

$$
\Delta\left(C_{j}, C_{k}\right)<\frac{4 r}{\beta^{\prime} r}=\delta
$$

which contradicts (3), and therefore $\nu=1$. Note that in this case $\beta^{\prime}$ depends only on $\delta$.

Now assume that (4) holds and let $\beta^{\prime}=7$. For each $k$ such that $C_{k}$ intersects $B(p, 2 r)$ and $\operatorname{diam} C_{k}>\beta^{\prime} r$, the corresponding $D_{k}$ is not contained in $B(p, 3 r)$. Let $B_{k}=B(q, r)$ be a disc centered at $q \in D_{k} \cap B(p, 2 r)$. Then $B_{k}$ is contained in $B(p, 3 r)$, and hence does not contain $D_{k}$. Therefore by (4) we have

$$
\operatorname{area}\left(D_{k} \cap B_{k}\right) \geq \tau r^{2} .
$$

Since any two distinct $D_{j}$ and $D_{k}$ are disjoint and all $B_{k}$ 's are contained in $B(p, 3 r)$, for the number $\nu$ of "large" $C_{k}$ 's as above we have

$$
\nu \tau r^{2} \leq \sum_{k} \operatorname{area}\left(D_{k} \cap B_{k}\right) \leq \operatorname{area}(B(p, 3 r))=9 \pi r^{2} .
$$

This gives $\nu \leq 9 \pi / \tau$, a bound that depends only on $\tau$.

Thus, to prove the existence of $\gamma_{2}$ it is enough to find a bound on the number of $C_{k}$ 's that intersect a given disc in $2 \mathcal{C}$ and satisfy

$$
\frac{1}{\beta} r \leq \operatorname{diam} C_{k} \leq \beta^{\prime} r
$$

Recall that by (1) there exists $\alpha \geq 1$ such that for any $C_{k}$ there exist inscribed and circumscribed concentric circles of radii $r_{k}$ and $R_{k}$, respectively, with $R_{k} / r_{k} \leq \alpha$. 
Since the interiors $D_{k}$ 's of distinct $C_{k}$ 's are disjoint, the corresponding inscribed discs are disjoint. Moreover, for $C_{k}$ satisfying (8) we have the following inequalities:

$$
\frac{1}{\beta} r \leq \operatorname{diam} C_{k} \leq 2 R_{k} \leq 2 \alpha r_{k} \leq \alpha \operatorname{diam} C_{k} \leq \alpha \beta^{\prime} r
$$

The number of curves $C_{k}$ that intersect a given disc $B(p, 2 r)$ in $2 \mathcal{C}$ is bounded above by the number of corresponding circumscribed discs that intersect the same disc $B(p, 2 r)$. Therefore, to find a bound $\gamma_{2}$ in (7) we need to find an upper bound on the number of disjoint open discs $B\left(p_{k}, r_{k}\right)$ of radii $r_{k}$ at least $r /(2 \alpha \beta)$ and at most $\beta^{\prime} r / 2$ such that $B\left(p_{k}, \alpha r_{k}\right)$ intersects $B(p, 2 r)$ (recall that $\left.R_{k} \leq \alpha r_{k}\right)$. Rescaling the Euclidean metric by $1 / r$ as above, it is equivalent to finding an upper bound on the number $n$ of disjoint open discs $B\left(p_{k}, r_{k}\right)$ of radii $r_{k}$ with $1 /(2 \alpha \beta) \leq r_{k} \leq \beta^{\prime} / 2$ such that $B\left(p_{k}, \alpha r_{k}\right)$ intersects $B(p, 2)$. Similar to the proof of $(6)$, each disc $B\left(p_{k}, \alpha r_{k}\right)$ intersecting $B(p, 2)$ is contained in $B\left(p, 2+\alpha \beta^{\prime}\right)$. Since $B\left(p_{k}, r_{k}\right)$ 's are disjoint, we obtain

$$
\sum_{i=1}^{n} r_{k}^{2} \leq\left(2+\alpha \beta^{\prime}\right)^{2}
$$

On the other hand,

$$
\sum_{i=1}^{n} r_{k}^{2} \geq \frac{n}{(2 \alpha \beta)^{2}}
$$

and thus $n \leq\left(2 \alpha \beta\left(2+\alpha \beta^{\prime}\right)\right)^{2}$, which completes the proof of $(7)$.

Proof of Proposition 2. Let $\epsilon>0$ be arbitrary and let $\mathcal{C}$ be a maximal collection of disjoint open discs of radii $\epsilon$ centered on $\mathcal{S}$.

We first establish an upper bound for $n(\epsilon)$ in terms of the function $N(x)$. For that one can define a function $f$ from $\mathcal{C}$ to the set $\mathcal{A}$ of all $C_{k}$ 's with $\epsilon / \beta \leq \operatorname{diam} C_{k} \leq \beta \epsilon$ by assigning to each disc in $\mathcal{C}$ a $C_{k} \in \mathcal{A}$ intersecting the disc. Note that by (2) every disc in $\mathcal{C}$ intersects at least one $C_{k}$ from $\mathcal{A}$, so that $f$ is defined on the whole set $\mathcal{C}$. Also, given any $C_{k}$ with $\operatorname{diam} C_{k} \leq \beta \epsilon$, by (6), there are at most $\gamma_{1} \operatorname{discs}$ in $\mathcal{C}$ that intersect $C_{k}$. Recall that $n(\epsilon)$ is the number of elements in $\mathcal{C}$, and the number of elements in $\mathcal{A}$ is at most $N(\beta / \epsilon)$. Hence, comparing the sizes of $\mathcal{C}$ and $\mathcal{A}$ via $f: \mathcal{C} \longrightarrow \mathcal{A}$, we immediately obtain

$$
n(\epsilon) \leq \gamma_{1} N(\beta / \epsilon)
$$

Therefore,

$$
\operatorname{dim}_{\text {ubox }} \mathcal{S}=\limsup _{\epsilon \rightarrow 0} \frac{\log n(\epsilon)}{\log (1 / \epsilon)} \leq \limsup _{\epsilon \rightarrow 0} \frac{\log \left(\gamma_{1} N(\beta / \epsilon)\right)}{\log (1 / \epsilon)}=\limsup _{x \rightarrow \infty} \frac{\log N(x)}{\log x},
$$

and similarly,

$$
\operatorname{dim}_{\text {lbox }} \mathcal{S} \leq \liminf _{x \rightarrow \infty} \frac{\log N(x)}{\log x} .
$$

We now use an argument similar to the one in the preceding paragraph to obtain an upper bound for $N(x)$ in terms of $n(\epsilon)$. Namely, we define a function $g$ from the set $\mathcal{B}$ of all $C_{k}$ 's satisfying $\epsilon / \beta \leq \operatorname{diam} C_{k}$ to $2 \mathcal{C}$ by assigning to each $C_{k} \in \mathcal{B}$ a disc in $2 \mathcal{C}$ intersecting $C_{k}$. By the maximality of $\mathcal{C}$, the collection $2 \mathcal{C}$ covers $\mathcal{S}$ and hence $g$ is defined on the whole set $\mathcal{B}$. By $(7)$, given a disc $B(p, 2 \epsilon) \in 2 \mathcal{C}$ there are at most $\gamma_{2}$ curves $C_{k}$ from $\mathcal{B}$ intersecting $B(p, 2 \epsilon)$. Recall that $N(\beta / \epsilon)$ is the number of elements 
in $\mathcal{B}$ and $n(\epsilon)$ is the number of elements in $\mathcal{C}$, which is the same as the number of elements in $2 \mathcal{C}$. Hence, comparing the sizes of $\mathcal{B}$ and $2 \mathcal{C}$ via $g: \mathcal{B} \longrightarrow 2 \mathcal{C}$, we obtain

$$
N(\beta / \epsilon) \leq \gamma_{2} n(\epsilon) \text {. }
$$

Therefore,

$$
\operatorname{dim}_{\text {ubox }} \mathcal{S}=\limsup _{\epsilon \rightarrow 0} \frac{\log n(\epsilon)}{\log (1 / \epsilon)} \geq \limsup _{\epsilon \rightarrow 0} \frac{\log \left(N(\beta / \epsilon) / \gamma_{2}\right)}{\log (1 / \epsilon)}=\limsup _{x \rightarrow \infty} \frac{\log N(x)}{\log x},
$$

and likewise

$$
\operatorname{dim}_{\text {lbox }} \mathcal{S} \geq \liminf _{x \rightarrow \infty} \frac{\log N(x)}{\log x} .
$$

The desired equalities are thus proved.

We finish this section with the following general theorem whose proof follows the lines of [Bo82, p. 250] or [W77, p. 126, Theorem 3].

Theorem 4. Let $\mathcal{P}=\left\{C_{k}\right\}_{k \in \mathrm{N} \cup\{0\}}$ be a packing such that the associated residual set $\mathcal{S}$ satisfies (1). Then

where $E$ is the exponent of $\mathcal{P}$.

$$
\limsup _{x \rightarrow \infty} \frac{\log N(x)}{\log x}=E,
$$

Proof. Assume that $C_{k}$ 's are numbered in such a way that the sequence $\left\{\operatorname{diam} C_{k}\right\}$ is monotone decreasing. The limit of this sequence must be 0 because of (1). Denote $r_{k}=\operatorname{diam} C_{k}$ and

$$
M=\limsup _{x \rightarrow \infty} \frac{\log N(x)}{\log x} .
$$

If $t>E$, then $\sum_{k} r_{k}^{t}$ is convergent and for any $x \in \mathbf{R}$ we have

$$
x^{-t} N(x) \leq \sum_{k} r_{k}^{t}<\infty .
$$

Taking $\log$ and the upper limit when $x \rightarrow \infty$, and then letting $t \rightarrow E_{+}$, we get $M \leq$ $E$. Assume for the sake of contradiction that $M<E$ and let $t$ satisfy $M<t<E$. Note that there exists $K \in \mathbf{R}$ such that for any $x \geq K$ we have

$$
t \geq \frac{\log N(x)}{\log x}
$$

Also, there exists $L \in \mathbf{N}$ such that for any $k \geq L$ and $x$ with $r_{k}^{-1} \leq x<r_{k+1}^{-1}$ we have $x \geq K$. Then $N(x)=k$, hence

$$
t \geq \frac{\log N(x)}{\log x}>\frac{\log k}{\log r_{k+1}^{-1}}
$$

and therefore, $r_{k+1}^{t}<k^{-1}$. Let $t^{\prime}$ satisfy $t<t^{\prime}<E$. Then

$$
r_{k+1}^{t^{\prime}}=\left(r_{k+1}^{t}\right)^{t^{\prime} / t}<k^{-t^{\prime} / t}
$$

for any $k \geq L$. Since $t^{\prime} / t>1, \sum_{k} r_{k}^{t^{\prime}}$ is a convergent series. This contradicts the definition of $E$.

Thus, for homogeneous residual sets we obtain the following analogue of Theorem 1: 
Corollary 5. If the residual set $\mathcal{S}$ of a packing is homogeneous and

$$
\operatorname{dim}_{\text {lbox }} \mathcal{S}=\operatorname{dim}_{\text {ubox }} \mathcal{S}=\operatorname{dim}_{H} \mathcal{S},
$$

then the limit of $\log N(x) / \log x$ as $x \rightarrow \infty$ exists and

$$
\lim _{x \rightarrow \infty} \frac{\log N(x)}{\log x}=E=\operatorname{dim}_{H} \mathcal{S} .
$$

Remark 4. There exist conditions on fractal sets that are easy to verify and that imply (9) (see, e.g., conditions in Theorems 3 and 4 in [F89]). In the next section we discuss another example of a homogeneous set satisfying (9), namely a Sierpiński carpet that is the Julia set of a hyperbolic rational map.

\section{Julia sets of hyperbolic rational maps}

In this section we prove an analogue of Theorem 1 for Sierpiński carpets that are Julia sets of hyperbolic rational maps (Theorem 6 below). Our proof is based on the application of Corollary 5 to Julia sets under consideration. For the background on the topics of complex dynamics used in this section see, e.g., [Be91, CG93, Mi06].

Let $f$ be a rational map of the Riemann sphere $\hat{\mathbf{C}}$. The Fatou set $\mathcal{F}(f)$ of $f$ is the set of all points $p$ in $\hat{\mathbf{C}}$ such that the family of iterates $\left\{f^{k}\right\}$ of $f$ is a normal family in some neighborhood of $p$. The Julia set $\mathcal{J}(f)$ is the complement of the Fatou set in $\hat{\mathbf{C}}$. From the definitions one immediately sees that $\mathcal{F}(f)$ is open and $\mathcal{J}(f)$ is compact. Moreover, $\mathcal{F}(f)$ and $\mathcal{J}(f)$ are completely invariant with respect to $f$, i.e.,

$$
f(\mathcal{F}(f))=f^{-1}(\mathcal{F}(f))=\mathcal{F}(f), \quad f(\mathcal{J}(f))=f^{-1}(\mathcal{J}(f))=\mathcal{J}(f) .
$$

If the Julia set $\mathcal{J}(f)$ of a rational map $f$ is a carpet (in which case we will refer to $\mathcal{J}(f)$ as the carpet Julia set $\mathcal{J}(f))$, then $\mathcal{J}(f)$ is the residual set associated to the packing formed by the connected components of the Fatou set $\mathcal{F}(f)=\bigcup_{k \in \mathrm{N} \cup\{0\}} D_{k}$. This follows from the definition of a residual set and the facts that $\mathcal{F}(f)$ is open, $\mathcal{J}(f) \cap \mathcal{F}(f)=\emptyset$, and $\mathcal{J}(f) \cup \mathcal{F}(f)=\hat{\mathbf{C}}$. The boundary curves $C_{k}=\partial D_{k}$ of these components are the peripheral circles of the carpet Julia set $\mathcal{J}(f)$.

A rational map $f$ is said to be hyperbolic if it is expanding in a neighborhood of its Julia set $\mathcal{J}(f)$ with respect to some conformal metric. More precisely, there exist a neighborhood $U$ of $\mathcal{J}(f)$, a smooth function $\lambda: U \rightarrow(0, \infty)$, and a constant $\rho>1$ such that

$$
\left\|f^{\prime}(z)\right\|_{\lambda}:=\frac{\lambda(f(z)) \cdot\left\|f^{\prime}(z)\right\|}{\lambda(z)} \geq \rho, \quad \forall z \in U
$$

where $\left\|f^{\prime}(z)\right\|$ denotes the spherical derivative of $f$ at $z$. In what follows we will only consider Julia sets that are not equal to the whole sphere. Therefore, we may assume that they are compact subsets of the plane and the spherical derivative in (10) can be replaced by the modulus of the derivative of $f$.

The function $\lambda$ can be used to define a new metric in a neighborhood of $\mathcal{J}(f)$ as follows. For a rectifiable path $\gamma$ in $U$ let

$$
\operatorname{length}_{\lambda}(\gamma):=\int_{\gamma} \lambda d s
$$


From (10) we immediately get

$$
\operatorname{length}_{\lambda}(f \circ \gamma) \geq \rho \operatorname{length}_{\lambda}(\gamma)
$$

for any rectifiable path $\gamma$ in $U$ such that $f \circ \gamma$ is also in $U$. Therefore, a hyperbolic rational map $f$ is locally expanding by a factor at least $\rho$ with respect to the path metric defined in a small neighborhood of $\mathcal{J}(f)$ by the formula

$$
d_{\lambda}(z, w):=\inf _{\gamma} \operatorname{length}_{\lambda}(\gamma)
$$

where $\gamma$ runs through all the rectifiable paths in $U$ connecting $z$ and $w$. Below we drop the index $\lambda$ and write $d(z, w)$ instead of $d_{\lambda}(z, w)$. Also, in what follows diam refers to the diameter with respect to $d_{\lambda}$. Since $\lambda$ is bounded away from 0 and $\infty$ in a neighborhood of $\mathcal{J}(f)$, the metric $d_{\lambda}$ is locally comparable (i.e., bi-Lipschitz equivalent) to the Euclidean metric in such a neighborhood. Therefore, it is irrelevant which metric, the Euclidean metric or the metric $d_{\lambda}$, one uses for verification of (1), $(2)$, and (3).

Theorem 6. Assume that $f$ is a hyperbolic rational map whose Julia set $\mathcal{J}(f)$ is a Sierpiński carpet. Then

$$
\lim _{x \rightarrow \infty} \frac{\log N(x)}{\log x}=E=\operatorname{dim}_{H} \mathcal{J}(f),
$$

where $N$ is the curvature distribution function and $E$ is the exponent of the packing by the connected components of the Fatou set of $f$, and $\operatorname{dim}_{H} \mathcal{J}(f)$ is the Hausdorff dimension of $\mathcal{J}(f)$.

Proof. We first show that if $f$ is a hyperbolic rational map whose Julia set $\mathcal{J}(f)$ is a Sierpiński carpet, then $\mathcal{J}(f)$ is homogeneous.

According to [F89, (3.6)], for a small enough $r>0$ and a ball $B(p, r)$ centered at $p \in \mathcal{J}(f)$ there exists a natural number $n$ so that the iterate $\left.f^{n}\right|_{B(p, r)}$ is bi-Lipschitz when the metric on $B(p, r)$ is rescaled by $1 / r$. More precisely, there exist positive constants $a, b, r_{0}$ such that for every $p \in \mathcal{J}(f)$ and $0<r \leq r_{0}$ there exists $n \in \mathbf{N}$ with

$$
a \frac{d(z, w)}{r} \leq d\left(f^{n}(z), f^{n}(w)\right) \leq b \frac{d(z, w)}{r}, \quad \forall z, w \in B(p, r)
$$

Note that condition (11) means that the Julia set $\mathcal{J}(f)$ of a hyperbolic rational map $f$ is approximately self-similar according to Definition 3.11 in [BK13]. Such approximate self-similarity is also discussed in [S83, p. 742]. In particular, [S83, Theorem 4] states that, up to a constant, the Hausdorff $\operatorname{dim}_{H} \mathcal{J}(f)$-measure is the unique conformal measure on $\mathcal{J}(f)$.

Now we are ready to prove that $\mathcal{J}(f)$ is homogeneous, namely it satisfies (1), (2), and (3). Let $\left\{C_{k}\right\}$ denote the sequence of peripheral circles of $\mathcal{J}(f)$. First of all, according to Whyburn's characterization [W58] we have $\operatorname{diam} C_{k} \rightarrow 0$ as $k \rightarrow \infty$. Also, since every peripheral circle $C$ of $\mathcal{J}(f)$ is the boundary of a connected component of $\mathcal{F}(f)$, the curve $f(C)$ is also a peripheral circle of $\mathcal{J}(f)$.

To show (1) assume that $C$ is an arbitrary peripheral circle of $\mathcal{J}(f)$ whose diameter is at most $\frac{a}{b} \min \left(r_{0}, a\right)$. We set $r=\frac{b}{a} \operatorname{diam} C$ and choose an arbitrary $p \in C$. Note that $C \subset B(p, r)$, since we may assume that $b / a>1$. Because $r \leq r_{0}$ and 
$p \in \mathcal{J}(f)$, by (11) there exists $n \in \mathbf{N}$ such that

$$
\frac{a^{2}}{b} \leq \operatorname{diam} f^{n}(C) \leq a .
$$

Since there are finitely many peripheral circles whose diameter is at least $\frac{a}{b} \min \left(r_{0}, a\right)$, there exists a constant $\alpha \geq 1$ such that for each of these "large" curves $C_{k}$ there exist inscribed and circumscribed concentric circles centered at $p_{k}$ of radii $r_{k}$ and $R_{k}$, respectively, with $R_{k} / r_{k} \leq \alpha$. Due to (12) the peripheral circle $f^{n}(C)$ is one of the large peripheral circles, say $C_{k}$. Let $q \in B(p, r)$ satisfy $f^{n}(q)=p_{k}$. Now (11) applied to $B(p, r)$ with $z=q$ and $w \in C$ implies that a disc centered at $q$ of radius at least $r r_{k} / b$ can be inscribed in $C$ and a disc centered at $q$ of radius at most $r R_{k} / a$ can be circumscribed around $C$. Therefore, the quotient of the circumscribed radius to the inscribed one for $C$ is at most $b \alpha / a$ and (1) follows.

We argue similarly to prove (2). Suppose that $p$ is an arbitrary point in $\mathcal{J}(f)$ and $0<r \leq \operatorname{diam} \mathcal{J}(f)$. If $r \geq \min \left(r_{0}, a / 2\right)$, then the fact that $\mathcal{J}(f)$ is a compact set with no interior points implies the existence of $\tilde{\beta}$ such that any disc $B(p, r)$ intersects a peripheral circle $C_{k}$ of $\mathcal{J}(f)$ with

$$
\frac{r}{\tilde{\beta}} \leq \operatorname{diam} C_{k} \leq \tilde{\beta} r .
$$

Moreover, by choosing $\tilde{\beta}$ sufficiently large, we can require that the diameter of each such $C_{k}$ is at most $a / 2$.

We now assume that $r<\min \left(r_{0}, a / 2\right)$. By the first inequality in (11), there exists $n \in \mathbf{N}$ such that $f^{n}(B(p, r))$ contains an open disc centered at $f^{n}(p)$ of radius at least $a$. It follows from the above that $B\left(f^{n}(p), a / 2\right)$ intersects a peripheral circle $C_{k}$ of $\mathcal{J}(f)$ with

$$
\frac{a}{2 \tilde{\beta}} \leq \operatorname{diam} C_{k} \leq \frac{a}{2}
$$

In particular, $C_{k}$ is contained in $B\left(f^{n}(p), a\right)$ and, consequently, in $f^{n}(B(p, r))$. Therefore, there exists a peripheral circle $C$ of $\mathcal{J}(f)$ that intersects $B(p, r)$ and such that $f^{n}(C)=C_{k}$. Moreover, (11) combined with (13) yield

$$
\frac{a}{2 b \tilde{\beta}} r \leq \operatorname{diam} C \leq \frac{1}{2} r
$$

The proof of $(2)$ (with $\beta=\max (1 / 2, \tilde{\beta}, 2 b \tilde{\beta} / a)$ ) is thus complete.

Finally, to prove (3) we argue by contradiction. Assume that there exists a sequence of pairs $\left\{C_{j_{i}}, C_{k_{i}}\right\}_{i}$ of distinct peripheral circles of $\mathcal{J}(f)$ such that $\Delta\left(C_{j_{i}}, C_{k_{i}}\right)$ $\rightarrow 0$ as $i \rightarrow \infty$. By symmetry we may assume that $\operatorname{diam} C_{j_{i}} \leq \operatorname{diam} C_{k_{i}}$ for all $i$. Since there are only finitely many peripheral circles of diameter at least a given number, we must have $\operatorname{diam} C_{j_{i}} \rightarrow 0$ as $i \rightarrow \infty$. Also,

$$
\operatorname{dist}\left(C_{j_{i}}, C_{k_{i}}\right)=\Delta\left(C_{j_{i}}, C_{k_{i}}\right) \operatorname{diam} C_{j_{i}} \rightarrow 0, \quad i \rightarrow \infty .
$$

Let $p_{i}$ be a point on $C_{j_{i}} \subset \mathcal{J}(f)$ and let $q_{i}$ be a point on $C_{k_{i}}$ such that

$$
\operatorname{dist}\left(C_{j_{i}}, C_{k_{i}}\right)=d\left(p_{i}, q_{i}\right) \text {. }
$$

Let $0<\epsilon<1$ be arbitrary and let $i$ be so large that

$$
r:=2 \operatorname{diam} C_{j_{i}} \leq r_{0} \quad \text { and } \quad \Delta\left(C_{j_{i}}, C_{k_{i}}\right)<\epsilon .
$$


Then $d\left(p_{i}, q_{i}\right)<r / 2$, hence $q_{i} \in B\left(p_{i}, r\right)$ and there is at least one more point $q^{\prime} \neq q_{i}$ in the intersection $C_{k_{i}} \cap B\left(p_{i}, r\right)$. By applying (11) to $B\left(p_{i}, r\right)$ with $z, w \in C_{j_{i}} \subset$ $B\left(p_{i}, r\right)$, we conclude that there exists $n \in \mathbf{N}$ such that for the peripheral circle $C_{j}=f^{n}\left(C_{j_{i}}\right)$ we have $\operatorname{diam} C_{j} \geq a / 2$. Similarly, by applying (11) to $B\left(p_{i}, r\right)$ with $z=q^{\prime}$ and $w=q_{i}$, we conclude that for the peripheral circle $C_{k}=f^{n}\left(C_{k_{i}}\right)$ we have $\operatorname{diam} C_{k} \geq a / 2\left(\right.$ recall that $\operatorname{diam} C_{k_{i}} \geq \operatorname{diam} C_{j_{i}}$ ). Finally, (11) applied to $B\left(p_{i}, r\right)$ with $z=p_{i}$ and $w=q_{i}$ gives

$$
\Delta\left(C_{j}, C_{k}\right) \leq 2 b \frac{\operatorname{dist}\left(C_{j_{i}}, C_{k_{i}}\right)}{r a}=2 b \frac{\Delta\left(C_{j_{i}}, C_{k_{i}}\right) \operatorname{diam} C_{j_{i}}}{r a}=\frac{b}{a} \Delta\left(C_{j_{i}}, C_{k_{i}}\right)<\frac{b}{a} \epsilon .
$$

This is a contradiction because $\epsilon$ is arbitrary and there are only finitely many pairwise disjoint peripheral circles $C$ with $\operatorname{diam} C \geq a / 2$. Hence, (3) follows.

The rest of the proof is a simple application of the results of [F89] and the previous results of this paper. Indeed, by [F89, Theorem in $\S 3]$, if $\mathcal{J}(f)$ is the Julia set of a hyperbolic rational map $f$, then

$$
\operatorname{dim}_{\text {lbox }} \mathcal{J}(f)=\operatorname{dim}_{\text {ubox }} \mathcal{J}(f)=\operatorname{dim}_{H} \mathcal{J}(f) .
$$

Corollary 5 therefore implies that the limit of $\log N(x) / \log x$ as $x \rightarrow \infty$ exists and

$$
\lim _{x \rightarrow \infty} \frac{\log N(x)}{\log x}=E=\operatorname{dim}_{H} \mathcal{J}(f) .
$$

This completes the proof of Theorem 6 .

Remark 5. The peripheral circles of $\mathcal{J}(f)$ as above are in fact uniform quasicircles, and hence $\mathcal{J}(f)$ also satisfies (4) (see [S95, Corollary 2.3]).

\section{Concluding remarks and further questions}

In their recent work [KO11] Kontorovich and Oh proved a result about Apollonian circle packings that strengthens Theorem 1. Namely, that $\lim _{x \rightarrow \infty}\left(N(x) / x^{\alpha}\right)=c$ for some positive constant $c$, where $\alpha=E=\operatorname{dim}_{H} \mathcal{S}$ (in the notation of Theorem 1). We believe that an analogue of this result also should hold in our context, i.e., for packings whose associated residual sets are homogeneous and approximately selfsimilar. More specifically, we make the following conjecture.

Conjecture 1. If $f$ is a hyperbolic rational map whose Julia set is a Sierpiński carpet, then there exists a positive constant $c$ such that

$$
N(x) \sim c \cdot x^{E}
$$

where $N$ is the curvature distribution function and $E$ is the exponent of the packing by the connected components of the Fatou set of $f$. In other words, $\lim _{x \rightarrow \infty}\left(N(x) / x^{E}\right)$ $=c$.

A homeomorphism $f:\left(X, d_{X}\right) \rightarrow\left(Y, d_{Y}\right)$ between two metric spaces is called quasisymmetric if there exists a homeomorphism $\eta:[0, \infty) \rightarrow[0, \infty)$ such that

$$
\frac{d_{Y}\left(f(x), f\left(x^{\prime}\right)\right)}{d_{Y}\left(f(x), f\left(x^{\prime \prime}\right)\right)} \leq \eta\left(\frac{d_{X}\left(x, x^{\prime}\right)}{d_{X}\left(x, x^{\prime \prime}\right)}\right)
$$

for any three distinct points $x, x^{\prime}$, and $x^{\prime \prime}$ in $X$. It turns out that quasisymmetric maps between subsets of the plane or the sphere preserve properties (1), (2), (3), and (4) up to a change of constants; see [H01] for background on quasisymmetric maps. An important invariant for quasisymmetric maps is the conformal dimension. 
For a metric space $\mathcal{S}$ this is the infimum of the Hausdorff dimensions of all images of $\mathcal{S}$ under quasisymmetric maps. The conformal dimension of a metric space is always at least its topological dimension and, trivially, at most the Hausdorff dimension. For example, the conformal dimension of the standard Cantor set $\mathcal{C}$ is zero, which is its topological dimension, and is strictly less than its Hausdorff dimension $\log 2 / \log 3$. The infimum in the definition of the conformal dimension of $\mathcal{C}$ is not achieved though, i.e., there is no metric space of Hausdorff dimension 0 that is quasisymmetric to $\mathcal{C}$.

The value of the conformal dimension of the Sierpiński carpet $S_{3}$ is unknown (see [H01, 15.22 Open problem]). However, it has a non-trivial lower bound $1+$ $\log 2 / \log 3$, which is strictly greater than its topological dimension 1 . If in the definition of conformal dimension we fix the target to be the plane, it is interesting to see what will be the shapes of the complementary components of quasisymmetric images of $S_{3}$ that are near-optimal with respect to the conformal dimension. Proposition 2 implies that the reciprocal diameters of peripheral circles of near-optimal images of $S_{3}$ must go to infinity faster than those for $S_{3}$. At present we do not know in what way the quasisymmetric map should distort the boundaries of the complementary components to make the Hausdorff dimension smaller.

\section{References}

[Be91] Beardon, A. F.: Iteration of rational functions. - Springer, New York, 1991.

[B97] Bishop, C. J.: Geometric exponents and Kleinian groups. - Invent. Math. 127:1, 1997, $33-50$.

[BK13] Bourdon, M., and B. Kleiner: Combinatorial modulus, the combinatorial Loewner property, and Coxeter groups. - Groups Geom. Dyn. 7:1, 2013, 39-107.

[BF11] Bourgain, J., and E. Fuchs: A proof of the positive density conjecture for integer Apollonian circle packings. - J. Amer. Math. Soc. 24:4, 2011, 945-967.

[Bo73] Boyd, D. W.: The residual set dimension of the Apollonian packing. - Mathematika 20, 1973, 170-174.

[Bo82] Boyd, D. W.: The sequence of radii of the Apollonian packing. - Math. Comp. 39:159, 1982, 249-254.

[CG93] Carleson, L., and Th. W. Gamelin: Complex dynamics. - Springer, New York, 1993.

[F89] Falconer, K. J.: Dimensions and measures of quasi self-similar sets. - Proc. Amer. Math. Soc. $106: 2,1989,543-554$.

[F14] FALCONER, K.: Fractal geometry. Mathematical foundations and applications. - John Wiley \& Sons, Ltd., Chichester, third edition, 2014.

[G03] Graham, R. L., J. C. Lagarias, C. L. Mallows, A. R. Wilks, and C. H. Yan: Apollonian circle packings: number theory. - J. Number Theory 100:1, 2003, 1-45.

[G05] Graham, R. L., J. C. Lagarias, C. L. Mallows, A. R. Wilks, and C. H. Yan: Apollonian circle packings: geometry and group theory. I. The Apollonian group. - Discrete Comput. Geom. 34:4, 2005, 547-585.

[G06-1] Graham, R. L., J. C. Lagarias, C. L. Mallows, A. R. Wilks, and C. H. Yan: Apollonian circle packings: geometry and group theory. II. Super-Apollonian group and integral packings. - Discrete Comput. Geom. 35:1, 2006, 1-36.

[G06-2] Graham, R. L., J. C. Lagarias, C. L. Mallows, A. R. Wilks, and C. H. Yan: Apollonian circle packings: geometry and group theory. III. Higher dimensions. - Discrete Comput. Geom. 35:1, 2006, 37-72.

[H01] Heinonen, J.: Lectures on analysis on metric spaces. - Springer-Verlag, New York, 2001. 
[KK00] KAPOVICH, M., and B. KLEINER: Hyperbolic groups with low-dimensional boundary. Ann. Sci. École Norm. Sup. (4) 33:5, 2000, 647-669.

[KO11] Kontorovich, A., and H. OH: Apollonian circle packings and closed horospheres on hyperbolic 3-manifolds. - J. Amer. Math. Soc. 24:3, 2011, 603-648.

[Mc98] McMullen, C. T.: Hausdorff dimension and conformal dynamics. III. Computation of dimension. - Amer. J. Math. 120:4, 1998, 691-721.

[Mi06] Milnor, J.: Dynamics in one complex variable. - Princeton Univ. Press, Princeton, NJ, third edition, 2006.

[OS12] Оh, H., and N. ShaH: The asymptotic distribution of circles in the orbits of Kleinian groups. - Invent. Math. 187:1, 2012, 1-35.

[P95] PARKer, J. R.: Kleinian circle packings. - Topology 34:3, 1995, 489-496.

[S08] SARnaK, P.: Equidistribution and primes. - Astérisque 322, 2008, 225-240.

[S11] SARnaK, P.: Integral Apollonian packings. - Amer. Math. Monthly 118:4, 2011, 291-306.

[S95] Schramm, O.: Transboundary extremal length. - J. Anal. Math. 66, 1995, 307-329.

[S83] Sullivan, D.: Conformal dynamical systems. - In: Geometric dynamics (Rio de Janeiro, 1981), Lecture Notes in Math. 1007, Springer, Berlin, 1983, 725-752.

[W77] Wilker, J. B.: Sizing up a solid packing. - Period. Math. Hungar. (2) 8, 1977, 117-134.

[W58] Whyburn, G. T.: Topological characterization of the Sierpiński curve. - Fund. Math. 45, $1958,320-324$.

Received 23 October $2014 \bullet$ Accepted 28 January 2015 